\title{
Use of adaptive learning algorithms in linear position measurement applications
}

\author{
Andreas Voss, Axel Bartos \\ TE Connectivity Sensors Germany GmbH, Hauert 13, 44227 Dortmund \\ Andreas.Voss@te.com
}

\section{Summary:}

The advancing digitalization of manufacturing ("smart factories") will create a high demand of position sensors. Besides environmental monitoring, position sensing will play a key role in future production processes. In summary, position measurement needs to become modular and scalable to different sensing dimensions with demanding accuracy requirements. XMR (magneto-resistive) technologies can give answers to achieve adaptive sensor systems under the objective of finding an optimum application solution in terms of performance, size and price.

Keywords: magneto-resistive, linear measurement, adaptive learning, contactless, sensor array

\section{The system point of view}

Designed as a miniaturized sensor system, position sensors must be as simple as possible due to customer acceptance and yet cover many requirements in terms of measuring distance, accuracy and performance. Although by definition, magnets are part of the measurement system, the sensor should work with existing magnets. For this purpose, an adaptive learning algorithm at the end of the line test (EOL) or during commissioning at the customer site is required. Miniaturization can be achieved by integration (MR sensor + ASIC) or by a new technological approach, which makes it possible to save measuring points and thus sensors, while achieving the desired measurement accuracy. Tunnel magneto resistive (TMR) technology [1] promises this simplification, since complex signal amplification can be omitted, and the technology is ready for direct chipintegration on ASICs.

\section{Methodology of magnet movement meas- urement}

Moving a permanent magnet on a linear path along an xMR [2][3] sensor establishes a characteristic output curve which can be used for a determination of the magnet position. Fig. 1 shows all three components of the magnetic field created by a magnet moving parallel to the $z$-axis with a certain distance $\boldsymbol{d}$ of the magnet to the sensor in y-direction. It stands out, that the $y$-component will be a good choice to use for a position determination, as it is continuous and free of singularity from its maximum to minimum. Using a simple Look-Up-Table (LUT) approach will solve the position mapping for different magnet-shapes $(\boldsymbol{\varnothing}, \boldsymbol{h})$ and distances between sensor and magnet.

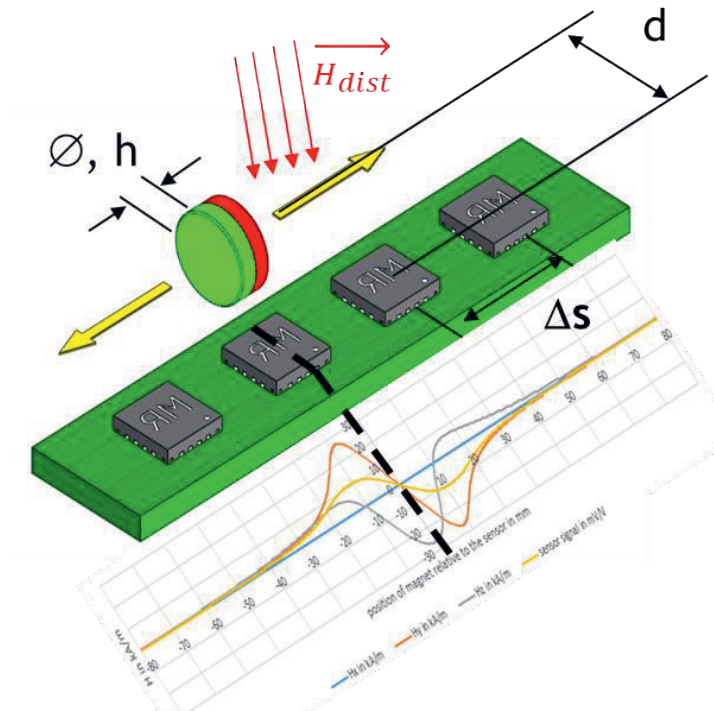

Fig. 1. Characteristic curve of magnet displacement along AMR sensor, y-component with continuous singularity-free function.

Although this single-sensor magnetic mapping only covers a short distance, it forms the basis of an absolute measurement system for the sensing of longer distances by being scalable and adaptable to customer requirements as part of a linear sensor kit [4].

\section{Absolute algorithm approach}

The logical next step is the arrangement of a series of sensors (see Fig. 1 , distance $\Delta \mathbf{s}$ ), which cover the entire magnetic movement.

When implementing an absolute measurement, only a single screenshot of the magnetic field distribution along the sensor array is possible. Now the whole characteristic sensor curve is populated with measurement points - one or two of them are situated in the linear region of the curve - all others are arranged at the left 


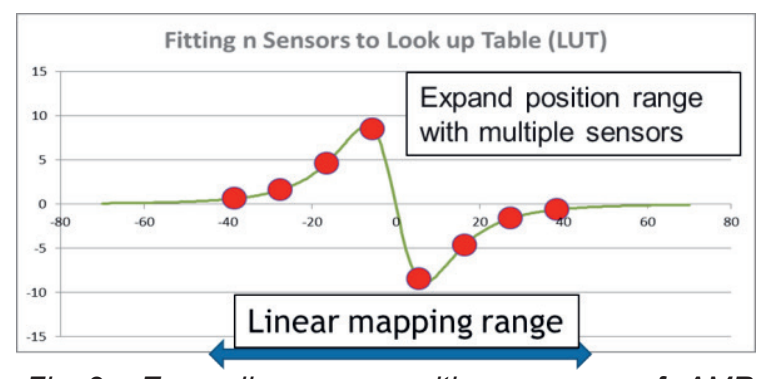

Fig. 2. Expanding range with an array of $A M R$ sensors.

and right section of the curve. A first approach is to find an algorithm, which determines the sensor(s) in the linear region and apply the LUT approach for one sensor described above. A smarter (and more robust) way is to include the available information from all sensors to determine the position. A closer look to Fig. 2 reveals a perfect match of the characteristic curve (green) with the single sensor signals (red dots) which will be the base of the now described procedure:

One algorithm, doing that fitting tas, $\mathrm{k}$ is the method of least squares (LS) [5], where the square of distances between all sensor node values $\boldsymbol{s}(\boldsymbol{n})$ and correspondent look-up-table values $\operatorname{LUT}[\boldsymbol{s}(\boldsymbol{n})]$ are summed up to find a local minimum, just where the LUT curve has its best match with the measured $\boldsymbol{m}$ sensor values as shown in the following formula:

$Q(\Sigma \rightarrow \operatorname{Min})=\operatorname{Min}\left(\sum_{n=1}^{m} \sqrt{\{s(n)-\operatorname{LUT}[s(n)]\}^{2}}\right)$

Runtime improvements can be achieved by using adaptive weighted averages [6] and golden ratio methods [7], resulting in up to 5 times faster position calculations than using formula (1) in stand-alone mode.

\section{Adaptive LUT learning}

Suppose to have a learning system, that can teach itself the LUT for position determination. This is very useful, when a standard sensor system needs to be adapted to a customer's application without HW changes. It requires a learning step with the application magnet, for example during the EOL Test. After the sensor installation, a full-scale movement of the magnet needs to be executed, while the sensor system is in learning mode. A prerequisite for this feature is to ensure that the movement is done with a constant velocity (which results in a constant $\Delta \boldsymbol{d}$ in Fig. 3). In this case the LUT learning algorithm can calculate the curve shape autonomously and normalizes the measured data as the real distance $\Delta s$ of the sensor elements on the hardware is known by design.

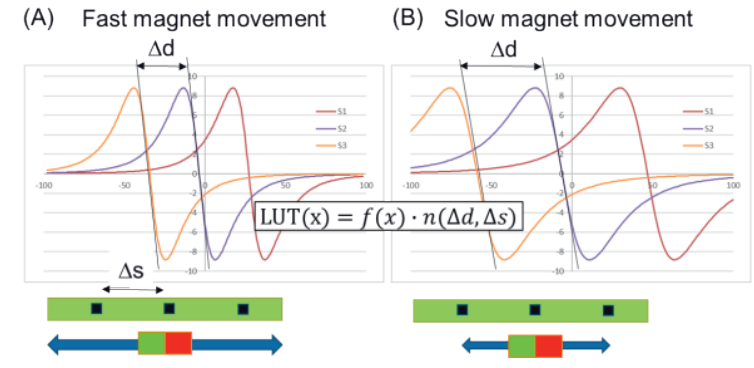

Fig. 3. Fast or slow (constant) magnet movement during learning step lead to real LUT.

A big advantage of this learning step is the possibility to map different magnets and inaccuracies of the magnet system in the LUT, as induced by e.g. slightly magnetic materials in the application housing or piston of hydraulic and pneumatic actuators.

\section{Outlook}

Since small sizes often offer an application advantage, sensor technologies are moving into focus which can make their contribution here. TMR will play a key role, as this technology provides an effect amplitude of approx. $1 \mathrm{Vpp}$ at $5 \mathrm{~V}$ supply. This allows TMR sensors to be wired directly to the ADC of an MCU or ASIC, opening the door to direct integration of both parts into one component housing. In combination with intelligent bus interfaces [8], the hardware simplification could lead to half of the system space needed today.

The future is found in a combination of intelligent adaptive software approaches to cover platform solutions which in best case could be adapted to a wide range of customer applications by simple customizing.

[1] M. Jullière: Tunneling between ferromagnetic films. In: Physics Letters A. Band 54, 1975, S. 225-226

[2] Thomson, W., "On the electrodynamic qualities of metall: effects on the electric conductivity of nickel and of iron", Proc. Roy. Soc. London, A8, 1857, S. 546-550

[3] R.Hunt, A Magnetoresistive Readout Transducer, IEEE Transactions on Magnetics 6(1):150 - $154 \cdot$ April 1971

[4] WO2011160796A3, US20170074715A1

[5] W.Press, S. Teukolsky, W. Vetterling, B. Flannery; Numerical Recipies in C: The Art of Scientific Computing, ISBN 0-521-43108-5, p.671

[6] N. Bronstein, K. A. Semendjajew u. a.: Taschenbuch der Mathematik. 2nd. edition. 1995, ISBN 9783817120086 p. 629

[7] Florian Jarre, Josef Stoer: Optimierung. Springer, Berlin 2004, ISBN 3-540-43575-1, S. $130 \mathrm{ff}$.

[8] DE102016002420A1 